\title{
A Comparative Study of Macular and Choroidal Thickness and Blood-Flow Parameters in Patients with Intermediate and Simple Juvenile Moderate Myopia
}

This article was published in the following Dove Press journal:

International Journal of General Medicine

\author{
TaiNan Lin ${ }^{1} * *$ \\ Yan Yang ${ }^{2, *}$ \\ JinHua Lin' \\ JiHui Zhang' \\ Qian Wen' \\ XiaoLu $\mathrm{He}^{\prime}$ \\ GuoQing Chen' \\ 'Department of Ophthalmology, Fujian \\ Provincial Governmental Hospital, \\ Fuzhou, 350003, People's Republic of \\ China; ${ }^{2}$ Fujian Center for Disease \\ Control and Prevention, Fuzhou, 35000I. \\ People's Republic of China \\ *These authors contributed equally to \\ this work
}

\begin{abstract}
Objective: This study aimed to compare the macular and choroidal thicknesses and bloodflow parameters of patients with intermediate and simple juvenile moderate myopia in order to provide a greater understanding of the pathogenesis of myopia and a basis for its prevention.

Methods: Participants were selected from patients under the age of 18 with moderate myopia who were treated in our ophthalmic clinic between June and December 2019. Seventy-five right eyes were selected from participants with a mean spherical equivalent ranging from -6.0 to $-3.0 \mathrm{D}$. These samples were divided into two groups based on eye axial length (AL). The thicknesses of the macula and choroid, the area of the foveal avascular zone (FAZ), and the blood flow density of the macular capillaries were measured, intergroup comparison was conducted.
\end{abstract}

Results: The average area of the FAZ was larger in the intermediate group than in the simple group. PERIM in the upper half was lower in the intermediate group than in the simple group, and the blood-flow density in the lower half of the macular area was higher in the simple group than in the intermediate group. The blood-flow density within $1 \mathrm{~mm}$ of the fovea centralis and the downward blood-flow density were higher in the intermediate group than in the simple group. The thicknesses of the lower part of the FAZ, the choroid of the fovea centralis, and the choroid under the retina were all larger in the intermediate group than in the simple group.

Conclusion: The area of the FAZ in patients with intermediate juvenile moderate myopia is larger than that in patients with simple myopia; the choroid in the fovea of macula compensatorily increases, and blood flow density also increases; the thickness of the choroid under the retina increases with myopia.

Keywords: choroidal blood flow, choroidal thickness, myopic adolescents, moderate myopia, optical coherence tomography angiography

\section{Introduction}

Since 2000, the prevalence of myopia has increased yearly, and juvenile myopia has become a more widespread global public health problem. ${ }^{1}$ The younger the age of an individual at the onset of myopia, the more likely it is to lead to high myopia in adulthood. $^{2}$ Cases with diopter values between -6.0 and $-3.0 \mathrm{D}$ are classed as moderate myopia, and simple and intermediate forms appear in almost half of all
Department of Ophthalmology, Fujian Provincial Governmental Hospital, No. 67 of Gupin Street, Gulou District, Fuzhou, 350003, People's Republic of China

Tel +8659188013047

Fax +8659188013003

Email lintainandr@163.com
International Journal of General Medicine 2021:14 1343-1348

1343

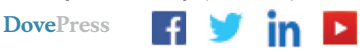

http://doi.org/10.2147/1/GM.S301702 
juvenile patients with moderate myopia. ${ }^{3}$ The axial length (AL) of an eye with simple myopia is between 22 and $25.5 \mathrm{~mm}$, while an $\mathrm{AL}$ of $\geq 25.5 \mathrm{~mm}$ represents the most common type of intermediate and pathological myopia. Moderate myopia, especially the intermediate form with an $\mathrm{AL}$ of $\geq 25.5 \mathrm{~mm}$, in children in the lower grades of elementary school is likely to lead to high myopia in adulthood. ${ }^{4}$ High myopia is a blinding eye disease that can lead to several serious complications, including complicated cataracts, glaucoma, myopic maculopathy, choroidal neovascularization, retinal detachment, retinal breaks, and posterior scleral staphyloma, all of which can impact a patient's quality of life. ${ }^{5}$ Therefore, more attention should be given to juvenile patients when they exhibit moderate myopia.

There are currently no studies on choroid and macular blood flow in moderate myopia. Optical coherence tomography angiography (OCTA), a recently developed clinical tool, is not sufficient for the accurate measurement of the deep perivascular microcirculation beyond the macular area, but it allows for non-invasive and quantitative studies of the retinal and choroidal vascular systems, including the microvascular-choroidal system. The present study analyzed the OCTA results of patients with moderate myopia with diopter values between -6.0 and $-3.0 \mathrm{D}$ and measured the thicknesses of the macula and choroid, the area of the foveal avascular zone (FAZ), and the blood-flow density of the macular capillaries. Subsequently, intergroup comparison was conducted, and the correlation of the findings with AL was analyzed. Finally, it was determined whether choroid and macular blood flow changes in intermediate myopia.

\section{Methods}

\section{Patients}

A total of 75 right eyes from 75 patients with moderate myopia (diopter values between -6.0 and $-3.0 \mathrm{D}$ ) were selected from patients under the age of 18 who had undergone treatment at our ophthalmic clinic between June 2019 and December 2019. The samples were divided into two groups based on $\mathrm{AL}$ : an intermediate group ( $\mathrm{AL} \geq$ $25.5 \mathrm{~mm} ; n=35)$ and a simple group (AL $<25.5 \mathrm{~mm}$, no pathological changes; $n=40$ ).

Exclusion criteria: (1) patients with other eye diseases, such as glaucoma, or any retinal vascular disease; (2) patients with a history of systemic diseases that may affect the circulation in the eye, such as diabetes; (3) patients with a history of intraocular surgery, laser photocoagulation, or eye injury.

This was a retrospective, cross-sectional, case-control study. All procedures were in accordance with the Declaration of Helsinki, and the Review Committee and Ethics Committee of our hospital approved the protocols used (approval no. 2019-03+11). All participants were fully informed of the purpose and procedures of the research.

\section{Data Collection}

Best corrected visual acuity testing, slit-lamp microscopy, optometry, intraocular pressure measurement, and corneal topography were conducted on all patients. OCTA (Zeiss Cirrus HD 5000A) was conducted based on the Fourier domain, with a light source wavelength of $840 \mathrm{~nm}$ and a maximum scanning speed of $70,000 / \mathrm{s}$. Each scan took approximately three seconds to complete. The retina scan mode was used to obtain retinal vascular density information. In this scanning mode, the angiograph scans the same area twice: the first scan consists of 13 horizontal scan lines with a width of $6 \mathrm{~mm}$ and 8 vertical scan lines with a width of $4 \mathrm{~mm}$, while the second scan consists of 13 vertical scan lines and 8 horizontal scan lines with a gap of $0.5 \mathrm{~mm}$ between each.

All examinations were performed by one experienced researcher, and each OCTA image was graded using the motion artifact score (MAS) (score 1-6). ${ }^{6}$ A higher MAS reflected more artifacts and lower image quality. Only the images with a MAS of $<2$ were used for analysis. The obtained En-Face images were then divided by the built-in software into their superficial retinal layer (SRL), which includes the retinal nerve fiber layer and retinal ganglion cell layer, and their deep retinal layer (DRL), which includes the retinal inner nuclear layer and retinal outer molecular layer, and the vascular density was calculated automatically. The main observation indexes (macular choroid thickness, FAZ area, and blood-flow density of the macular capillaries) were measured, inter-group comparison was conducted, and the correlation of the indexes with AL was analyzed.

\section{Statistical Analysis}

Data were statistically analyzed using statistical software SPSS 19.0. All data were expressed as mean \pm standard deviation. The inter-group comparison was conducted using one-way analysis of variance, and the inter-group pairwise comparison was conducted using the least 
significant difference method. The correlations between $\mathrm{AL}$, retinal thickness, and the density of the choroidal capillaries were analyzed using Kendall's tau (for nonnormally distributed data) or Spearman's rank correlation coefficient (for normally distributed data). A $P$-value of $<0.05$ was considered statistically significant.

\section{Results}

The differences in age $(F=0.68, P>0.05)$ and gender between the two groups were not statistically significant. The differences in mean spherical equivalent (MSE) $(F=$ 41.04, $P<0.001)$ and corneal curvature $(\mathrm{P}<0.05)$ were statistically significant. The age, MSE, corneal curvature, eye AL, FAZ area size, choroidal thickness in the macular area, choroidal thickness in the center concave, and retinal thickness of the patients in the two groups are shown in Table 1.

The FAZ area was larger in the intermediate group than in the simple group, and the difference was statistically significant. PERIM in the upper half was lower in the intermediate group than in the simple group, and the difference was statistically significant. The blood-flow density in the lower half of the image was significantly higher in the simple group than in the intermediate group. The blood-flow density within $1 \mathrm{~mm}$ of the fovea centralis was higher in the intermediate group than in the simple group, and the difference was statistically significant. The downward blood-flow density was significantly higher in the intermediate group than in the simple group. There were no significant differences between the two groups in the thickness of the FAZ of the whole image or in the upper half of the FAZ, but the thickness of the lower part of the FAZ was higher in the intermediate group than in the simple group, and the difference was statistically

Table I Baseline Information Between the Intermediate Myopia and the Simple Myopia Groups

\begin{tabular}{|l|c|c|c|}
\hline Characteristics & $\begin{array}{c}\text { The Intermediate } \\
\text { Myopia Group }\end{array}$ & $\begin{array}{c}\text { Simple } \\
\text { Myopia } \\
\text { Group }\end{array}$ & $p$ \\
\hline Gender (M:F) & $16: 18$ & $19: 17$ & 0.632 \\
\hline Age (yr) & $13.4 \pm 2.7$ & $14.4 \pm 2.8$ & 0.680 \\
\hline MSE (Diopter) & $-4.97 \pm 0.76$ & $-4.20 \pm 0.82$ & 0.001 \\
\hline AL (mm) & $26.13 \pm 0.58$ & $25.04 \pm 0.38$ & 0.000 \\
\hline $\begin{array}{l}\text { Corneal } \\
\text { curvature (mm) }\end{array}$ & $7.9 \pm 0.22$ & $7.79 \pm 0.25$ & 0.021 \\
\hline
\end{tabular}

significant. The choroid thickness of the fovea centralis was higher in the intermediate group than in the simple group, and the difference was statistically significant.

No significant differences were observed in the following measures: the thickness of the FAZ in the parafovea; the upper part of the FAZ and the lower part in the parafovea; the thickness of the upper part; temporal thickness; nasal thickness; lower thickness of the whole image; blood-flow density of the whole image; blood-flow density in the upper half; blood-flow density in the lower half of the macula; blood-flow density within $1 \mathrm{~mm}$ of the fovea centralis; blood-flow density in the parafovea; the upper or lower half; temporal blood-flow density; upward or downward blood-flow density; and nasal blood-flow density (Table 2).

In patients with moderate myopia, blood-flow density in the lower half of the macular area was negatively correlated with AL $(r=-0.243, P=0.043)$, while the blood-flow density of the fovea centralis was positively correlated with $\mathrm{AL}(r=0.310, P=0.009)$. There were no significant correlations between $\mathrm{AL}$ and total blood-flow density, the blood-flow density in the upper half of the macular area, or the blood-flow density in the parafovea $(P>0.05)$. Moreover, the thickness of the lower part of the FAZ was negatively correlated with $\mathrm{AL}(r=-0.277, P=$ 0.020 ), while the thickness of the choroid of the fovea centralis was positively correlated with AL $(r=0.226, P=$ 0.006). There were no significant correlations between $\mathrm{AL}$ and retinal thickness, the thickness of the upper or lower half of the parafovea, temporal thickness, upper thickness, or nasal thickness $(P>0.05)$.

\section{Discussion}

Intermediate myopia can lead to developmental disorders: growth in the later period is disproportionate, and the elongation of the $\mathrm{AL}$ is beyond the effective range of the neutralizing effect of the reduction in refractive power caused by the cornea and lens. ${ }^{7,8}$ Although it has no impact on visual function, uncontrolled moderate myopia is likely to result in high myopia, ${ }^{9}$ a blinding eye disease. It can also lead to a series of severe secondary complications, such as complicated cataracts, glaucoma, myopic maculopathy, choroidal neovascularization, retinal detachment, retinal breaks, and posterior scleral staphyloma, ${ }^{9,10}$ all of which can seriously impact vision and quality of life. Therefore, juvenile moderate myopia should be studied in depth. However, to date, there have been no studies on 
Table 2 Macular Choroid Thickness and Blood Flow Parameters Between the Intermediate Myopia and the Simple Myopia Groups

\begin{tabular}{|c|c|c|c|}
\hline Characteristics & The Intermediate Myopia Group & Simple Myopia Group & $p$ \\
\hline $\mathrm{FAZ}\left(\mathrm{mm}^{2}\right)$ & $0.68 \pm 0.81$ & $0.31 \pm 0.10$ & 0.000 \\
\hline PERIM $\left(\mathrm{mm}^{2}\right)$ & $1.76 \pm 0.47$ & $2.20 \pm 0.38$ & 0.000 \\
\hline Blood flow density throughout the image (\%) & $48.58 \pm 1.98$ & $47.43 \pm 2.94$ & 0.160 \\
\hline Upper half blood flow density (\%) & $48.81 \pm 2.31$ & $47.54 \pm 2.96$ & 0.052 \\
\hline Lower blood flow density (\%) & $41.46 \pm 13.39$ & $47.33 \pm 3.03$ & 0.011 \\
\hline Macular fovea Imm blood flow density (\%) & $27.21 \pm 13.49$ & $15.77 \pm 6.08$ & 0.000 \\
\hline Peripheral fovea blood flow density (\%) & $51.64 \pm 2.13$ & $50.78 \pm 3.29$ & 0.197 \\
\hline Temporal blood flow density (\%) & $50.52 \pm 1.79$ & $49.40 \pm 3.77$ & 0.122 \\
\hline Upper flow density (\%) & $52.84 \pm 2.81$ & $52.09 \pm 3.75$ & 0.348 \\
\hline Nosebleed density (\%) & $51.54 \pm 2.97$ & $50.10 \pm 3.20$ & 0.057 \\
\hline Lower blood flow density (\%) & $111.70 \pm 108.52$ & $51.53 \pm 3.54$ & 0.001 \\
\hline Overall image thickness $(\mu \mathrm{m})$ & $308.00 \pm 13.97$ & $307.58 \pm 9.15$ & 0.882 \\
\hline Half the thickness of the $(\mu \mathrm{m})$ & $306.35 \pm 26.86$ & $309.53 \pm 9.21$ & 0.506 \\
\hline The second half of the thickness $(\mu \mathrm{m})$ & $290.09 \pm 32.11$ & $305.64 \pm 9.67$ & 0.007 \\
\hline Macular fovea thickness $(\mu \mathrm{m})$ & $264.03 \pm 33.07$ & $241.22 \pm 15.88$ & 0.001 \\
\hline Thickness of adjacent fovea $(\mu \mathrm{m})$ & $317.44 \pm 17.52$ & $317.19 \pm 9.77$ & 0.933 \\
\hline Half thickness above side fovea $(\mu \mathrm{m})$ & $318.32 \pm 15.12$ & $318.97 \pm 9.54$ & 0.832 \\
\hline Half thickness of side fovea $(\mu \mathrm{m})$ & $313.06 \pm 15.46$ & $315.36 \pm 10.53$ & 0.467 \\
\hline The thickness of the temporal $(\mu \mathrm{m})$ & $311.53 \pm 14.42$ & $307.56 \pm 10.00$ & 0.183 \\
\hline Thickness of the upper part $(\mu \mathrm{m})$ & $322.00 \pm 15.97$ & $322.81 \pm 9.65$ & 0.798 \\
\hline Nasal thickness $(\mu \mathrm{m})$ & $321.59 \pm 16.74$ & $322.08 \pm 11.01$ & 0.884 \\
\hline Lower thickness $(\mu \mathrm{m})$ & $254.58 \pm|| 4.6 \mid$ & $316.42 \pm 10.81$ & 0.002 \\
\hline
\end{tabular}

choroidal thickness and blood-flow changes in the macular area of patients with juvenile moderate myopia.

In the present study, OCTA was used to measure the intraocular parameters of intermediate moderate myopia, including MSE, corneal curvature, eye AL, size of the FAZ area, choroidal thickness in the macular area, choroidal thickness in the center concave, and retinal thickness. It was found that the area of the FAZ was larger in cases of intermediate myopia than in simple myopia.

The macular center concave is surrounded by a capillary arch with an avascular area of a few hundred microns in the center-this area is the FAZ. The FAZ is essential for maintaining fine vision; when the axis of the eye becomes longer, making the retina thinner and affecting the macular area, the shape and size of the FAZ changes. ${ }^{11-13}$ The present study identified no differences between the two groups in the thickness of the overall parafovea or its upper or lower half, temporal thickness, upper thickness, or nasal thickness. Meanwhile, the blood flow and choroid thickness of the fovea centralis were higher in the intermediate group than in the simple group. This indicates that, in juveniles, the capillary density of the choroid in the fovea centralis increases and the choroid is thickened to adapt to the metabolism of the peripheral retina after axial lengthening. This finding is consistent with the results of studies conducted by Sung et al. ${ }^{14-16}$ During this adaptation, the choroid associated with the increased AL of the eye also presents compensatory thickening, and the blood-flow rate increases. As the AL increases, the thickness of the retina also increases to realize the blood supply around the retina. This indicates that the lengthening of the ocular axis may cause the compensatory thickening of the choroidal capillary layer in the fovea centralis and increase the number of capillaries and their volume. If the degree of myopia continues to increase, the AL of the eye will continue to do so as well. The retina may not be able to compensate accordingly, so the choroid and the capillary layer of the retina may become thinner. ${ }^{17-19}$

The choroidal thickness of the lower part was higher in the intermediate group than in the simple group, and the difference was statistically significant. No other choroidal diseases were found in the patients with intermediate myopia. In the intermediate group, the area with the thinnest choroidal layer was located at the bottom, but there were no differences in other directions between the two groups. This is consistent with the choroidal changes in adults with high myopia reported in other studies. ${ }^{20-23}$ 
However, due to the uneven distribution of the optic vertebrae cells, the metabolism requirement under the outer layer of the retina is reduced, ${ }^{24}$ making the choroidal thickness of the lower part thinner. Furthermore, the inferior choroid begins to close at 15 weeks in the fetal period, and the closed position of the fetal choroidal fissure is the weakest position of the whole choroid. ${ }^{25,26}$ Thus, with an increase in the degree of myopia and the extension of the ocular axis, the whole eye can expand, causing the bottom to become thinner first. This suggests that, during the lengthening of the ocular axis in juveniles, the area with the lowest choroidal thickness is located below, and no significant changes occur in the corresponding central area, temporal side, or upper part. As the choroid at this site is relatively weak, the thickness of the choroid is decreased there first when the eye axis becomes longer.

\section{Conclusion}

In the present study, clear imaging of the choroidal structure and reconstruction of the choroidal capillary layer were obtained using OCTA technology without a contrast agent. Choroid thickness and the density of choroidal capillaries were analyzed using post-processing. It was found that the area of the FAZ was larger in the intermediate group than in the simple group, that blood-flow density and the thickness of the choroid in the fovea of the macula increased, that the thickness of the choroid under the retina also increased with myopia, and that the axis of the eye showed changes earlier than other areas. These findings can be used to provide early intervention and in the creation of conditions to control the development of myopia.

\section{Ethics Approval and Consent to Participate}

This study was conducted in accordance with the declaration of Helsinki. This study was conducted with approval from the Ethics Committee of Fujian Provincial Governmental Hospital. A written informed consent was obtained from legal guardians of all participants.

\section{Consent for Publication}

Consent for publication was obtained from every individual (their parents/legal guardians) whose data are included in this manuscript.

\section{Acknowledgment}

Fund Projects: Fujian Health Innovation Project No:2019CX-13.

\section{Disclosure}

The authors report no conflicts of interest in this work.

\section{References}

1. Dolgin E. The myopia boom. Nature. 2015;519(7543):276-278. doi:10.1038/519276a

2. Xu L, Wang Y, Wang S, Wang Y, Jonas JB. High myopia and glaucoma susceptibility. The Beijing Eye Study. Ophthalmology. 2007;114(2):216-220. doi:10.1016/j.ophtha.2006.06.050

3. Shi YN, Fang Y. The mechanism, prediction, prevention and control of myopia among children and adolescents in China. Shaanxi Science \& Technology Press; 2012:P127-128.

4. He M, Du Y, Liu Q, et al. Effects of orthokeratology on the progression of low to moderate myopia in Chinese children. $B M C$ Ophthalmol. 2016;16(1):126. doi:10.1186/s12886-016-0302-5

5. Jonas JB, Gusek GC, Naumann GO. Optic disk morphometry in high myopia. Graefes Arch Clin Exp Ophthalmol. 2018;226(6):587-590. doi:10.1007/BF02169209

6. Cui Y, Zhu Y, Wang JC, et al. Imaging artifacts and segmentation errors with wide-field swept-source optical coherence tomography angiography in diabetic retinopathy. Transl Vis Sci Technol. 2019;8 (6): 18. doi:10.1167/tvst.8.6.18

7. Sanders DR, Vukich JA, Doney K, Gaston M. U.S. food and drug administration clinical trial of the implantable contact lens for moderate to high myopia. Ophthalmology. 2003;110(2):255-266. doi:10.1016/s0161-6420(02)01771-2

8. Hirata M, Tsujikawa A, Matsumoto A, et al. Macular choroidal thickness and volume in normal subjects measured by swept-source optical coherence tomography. Invest Ophthalmol Vis Sci. 2011;52 (8):4971-4978. doi:10.1167/iovs.11-7729

9. Lan YW, Chang SY, Sun FJ, Hsieh JW. Different disc characteristics associated with high myopia and the location of glaucomatous damage in primary open-angle glaucoma and normal-tension glaucoma. $J$ Glaucoma. 2019;28(6):519-528. doi:10.1097/ IJG.0000000000001217

10. Fledelius HC, Jacobsen N, Li XQ, Goldschmidt E. Choroidal thickness at age 66 years in the Danish high myopia study cohort 1948 compared with follow-up data on visual acuity over 40 years: a clinical update adding spectral domain optical coherence tomography. Acta Ophthalmol. 2018;96(1):46-50. doi:10.1111/ aos. 13659

11. Flores-Moreno I, Lugo F, Duker JS, Ruiz-Moreno JM. The relationship between axial length and choroidal thickness in eyes with high myopia. Am J Ophthalmol. 2013;155(2):314-319.e1. doi:10.1016/j. ajo.2012.07.015

12. Gupta P, Cheung CY, Saw S-M. Peripapillary choroidal thickness in young Asians with high myopia. Invest Ophthalmol Vis Sci. 2015;56 (3):1475-1481. doi:10.1167/iovs.14-15742

13. Wang NK, Lai CC, Chou CL, et al. Choroidal thickness and biometric markers for the screening of lacquer cracks in patients with high myopia. PLoS One. 2013;8(1):e53660. doi:10.1371/journal. pone. 0053660

14. Sung MS, Lee TH, Heo H, Park SW. Association between optic nerve head deformation and retinal microvasculature in high myopia. $A m$ J Ophthalmol. 2018;188:81-90. doi:10.1016/j.ajo.2018.01.033 
15. Tan CS, Lim LW, Chow VS, et al. Optical coherence tomography angiography evaluation of the parafoveal vasculature and its relationship with ocular factors. Invest Ophthalmol Vis Sci. 2016;57(9): OCT224-34. doi:10.1167/iovs.15-18869

16. Sayanagi K, Ikuno Y, Uematsu S, Nishida K. Features of the choriocapillaris in myopic maculopathy identified by optical coherence tomography angiography. $\quad B r \quad J \quad$ Ophthalmol. 2017;101 (11):1524-1529. doi:10.1136/bjophthalmol-2016-309628

17. Miyata M, Ooto S, Hata M, et al. Detection of myopic choroidal neovascularization using optical coherence tomography angiography. Am J Ophthalmol. 2016;165:108-114. doi:10.1016/j.ajo.2016.03.009

18. Louzada RN, Ferrara D, Novais EA, et al. Analysis of scleral feeder vessel in myopic choroidal neovascularization using optical coherence tomography angiography. Ophthalmic Surg Lasers Imaging Retina. 2016;47(10):960-964. doi:10.3928/23258160-20161004-11

19. Harb E, Hyman L, Gwiazda J, et al.; COMET Study Group. Choroidal thickness profiles in myopic eyes of young adults in the correction of myopia evaluation trial cohort. Am J Ophthalmol. 2015;160(1):62-71.e2. doi:10.1016/j.ajo.2015.04.018

20. Jiang R, Wang YX, Wei WB, Xu L, Jonas JB. Peripapillary choroidal thickness in adult Chinese: the Beijing eye study. Invest Ophthalmol Vis Sci. 2015;56(6):4045-4052. doi:10.1167/iovs.15-16521
21. Entezari M, Karimi S, Ramezani A, Nikkhah H, Fekri Y, Kheiri B. Choroidal thickness in healthy subjects. J Ophthalmic Vis Res. 2018;13(1):39-43. doi:10.4103/jovr.jovr_148_16

22. He XG, Deng JJ, Yin Y, et al. Macular choroidal thickness in Chinese preschool children: decrease with axial length but no evident change with age. Int J Ophthalmol. 2019;12(9):1465-1473. doi:10.18240/ ijo.2019.09.15

23. Chen LL, Chen ZL, Li ZB. Subfoveal choroidal thickness measured by Cirrus HD optical coherence tomography in myopia. Int J Ophthalmol. 2014;14(9):1641-1643.

24. Ikuno Y, Kawaguchi K, Nouchi T, Yasuno Y. Choroidal thickness in healthy Japanese subjects. Invest Ophthalmol Vis Sci. 2010;51 (4):2173-2176. doi:10.1167/iovs.09-4383

25. Read SA, Collins MJ, Vincent SJ, Alonso-Caneiro D. Choroidal thickness in childhood. Invest Ophthalmol Vis Sci. 2013;54 (5):3586-3593. doi:10.1167/iovs.13-11732

26. Ruiz-Moreno JM, Flores-Moreno I, Lugo F, Ruiz-Medrano J, Montero JA, Akiba M. Macular choroidal thickness in normal pediatric population measured by swept-source optical coherence tomography. Invest Ophthalmol Vis Sci. 2013;54(1):353-359. doi:10.1167/iovs.12-10863

\section{Publish your work in this journal}

The International Journal of General Medicine is an international, peer-reviewed open-access journal that focuses on general and internal medicine, pathogenesis, epidemiology, diagnosis, monitoring and treatment protocols. The journal is characterized by the rapid reporting of reviews, original research and clinical studies across all disease areas. The manuscript management system is completely online and includes a very quick and fair peer-review system, which is all easy to use. Visit http://www.dovepress.com/ testimonials.php to read real quotes from published authors. 